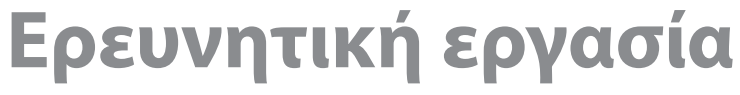 Research article
}

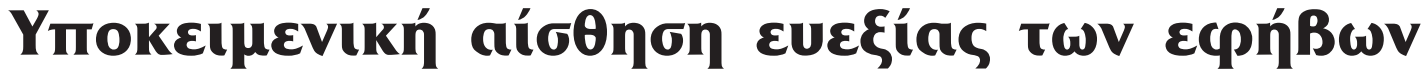 kal o póños tins olkoyéveias}

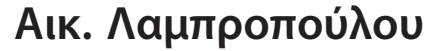

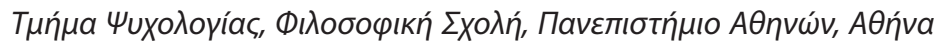

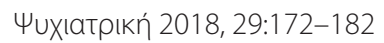

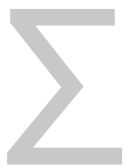

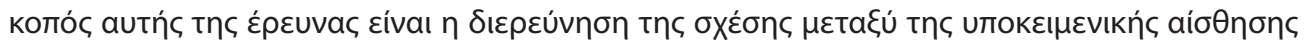

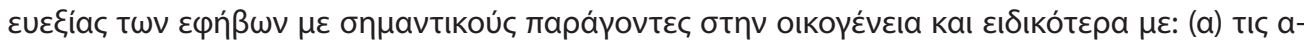

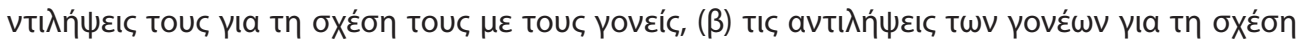

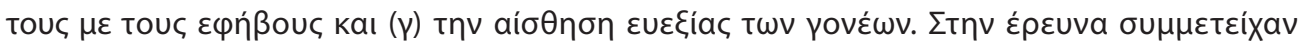

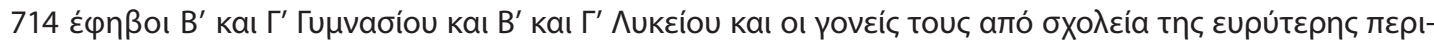

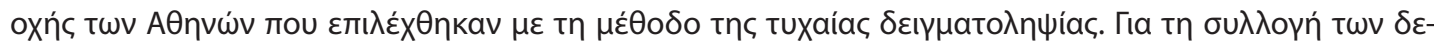

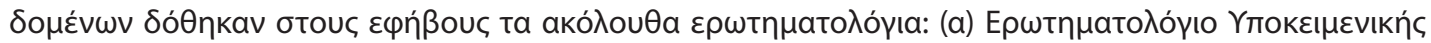

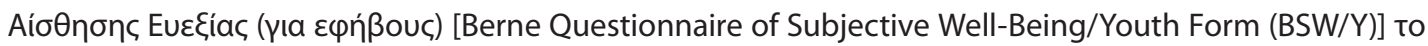

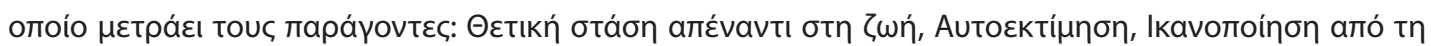

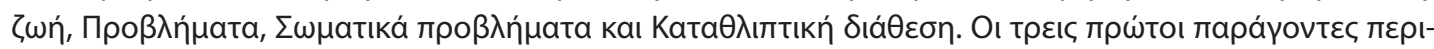

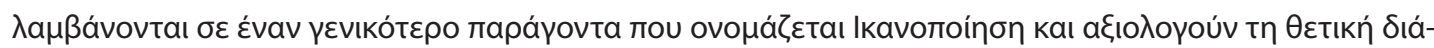

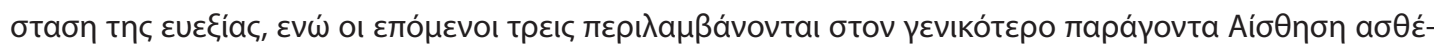

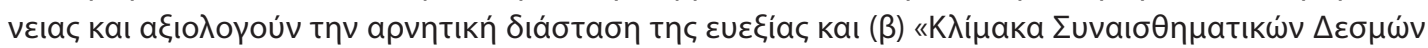

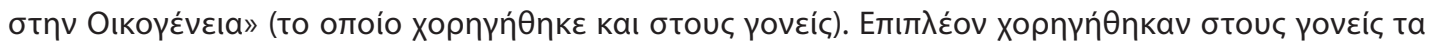

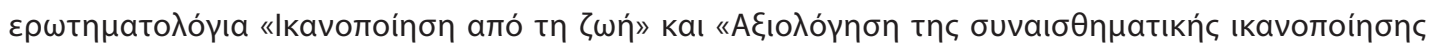

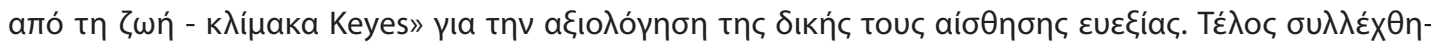

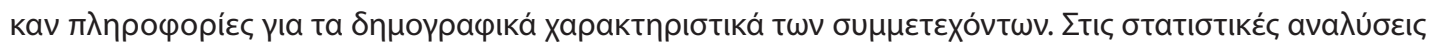

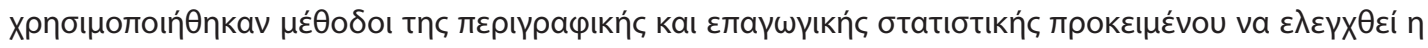

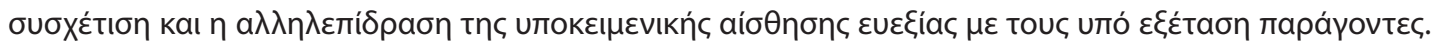

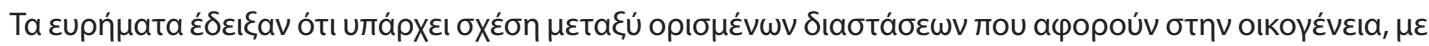

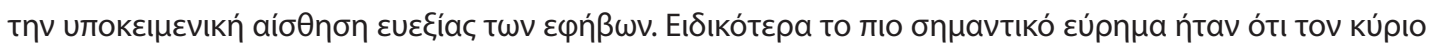

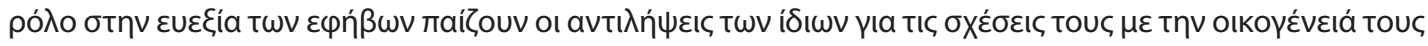

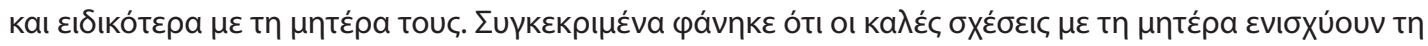

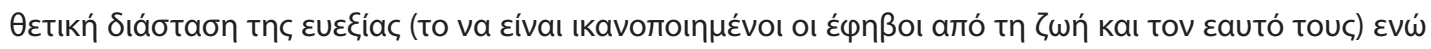




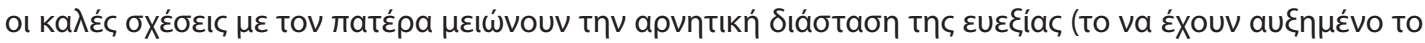

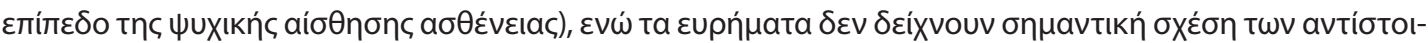

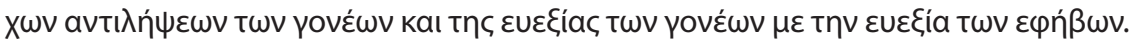

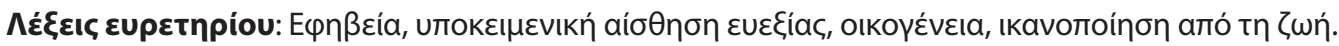

\section{Eıбaywyń}

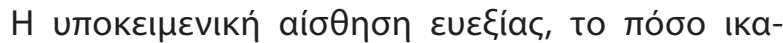

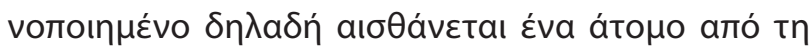

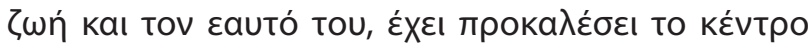

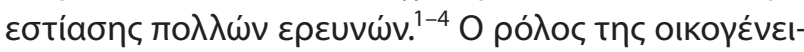

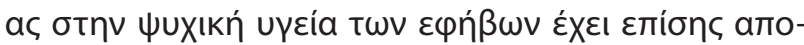

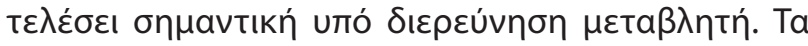

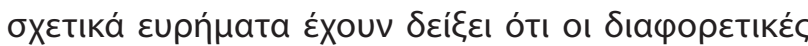

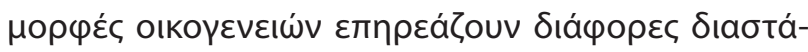

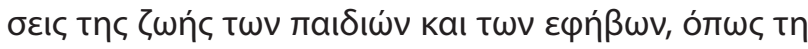

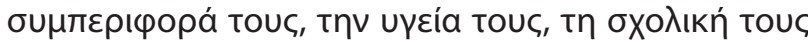

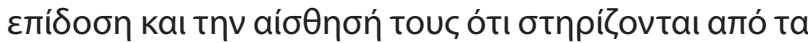

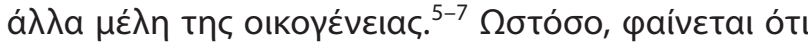

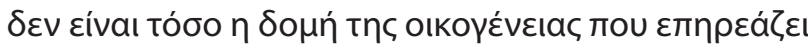

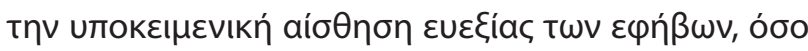

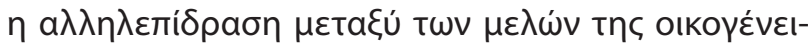

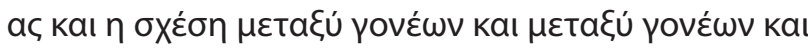
паı $\_\omega ́ v .8$

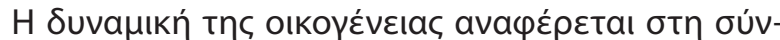

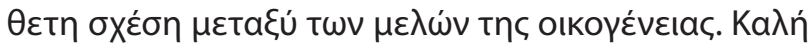

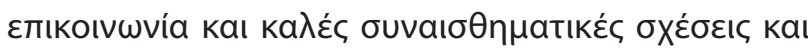

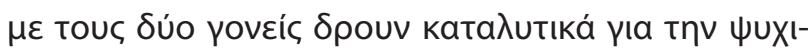

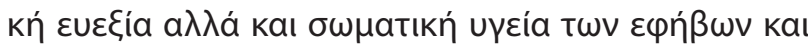

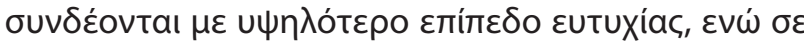

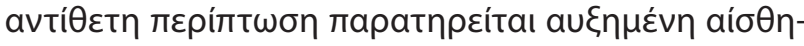

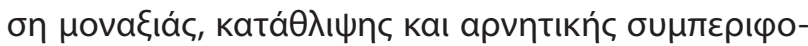
pác. 9,10

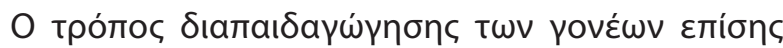

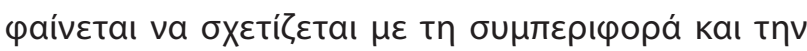

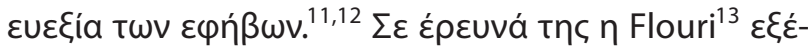

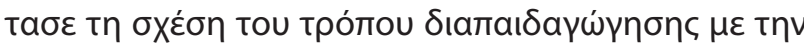

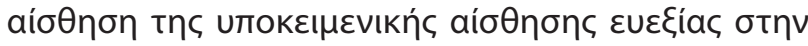

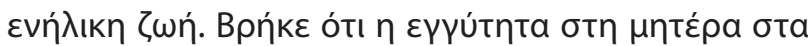

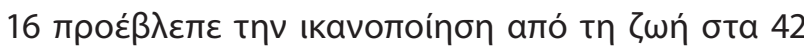

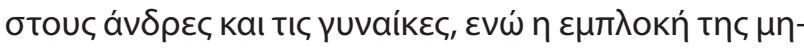

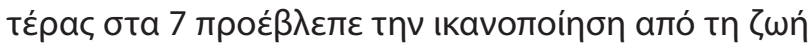

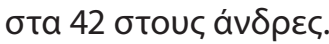

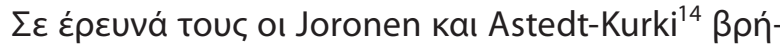

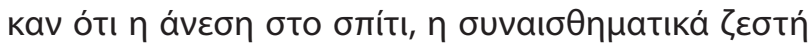

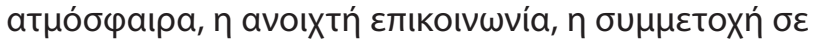

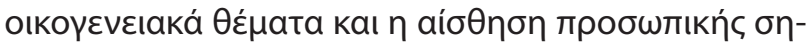

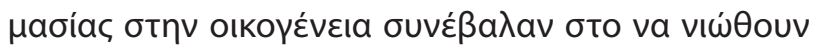

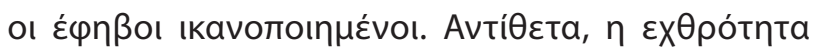

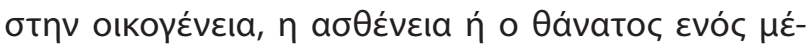

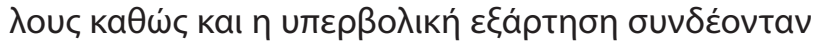

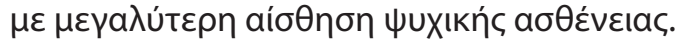

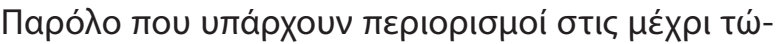

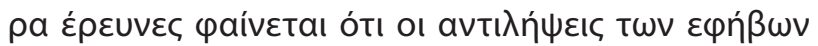

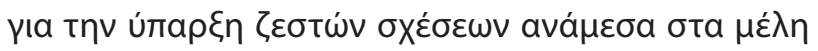

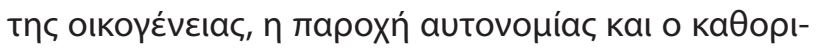

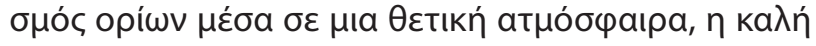

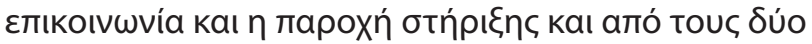

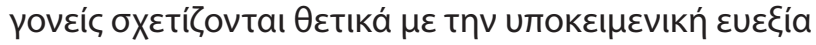
$\tau \omega v \varepsilon \varphi n ́ \beta \omega v$.

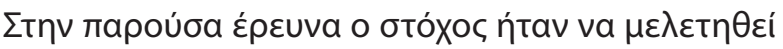

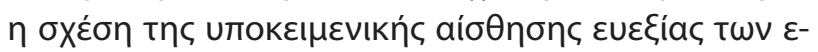

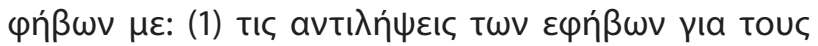

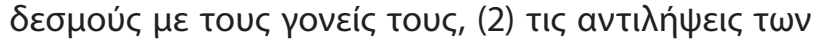

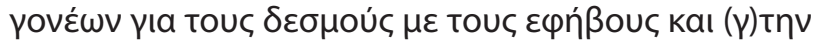

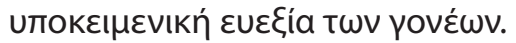

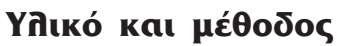

\section{$\Delta \varepsilon i ́ y \mu a$}

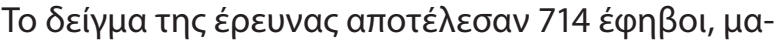

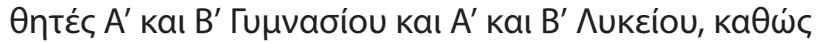

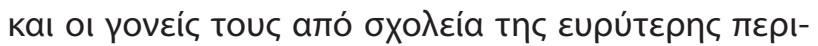

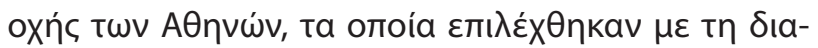

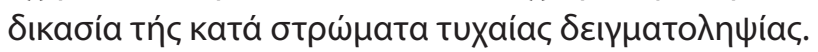

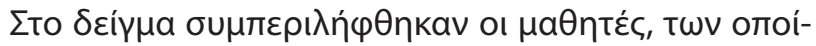

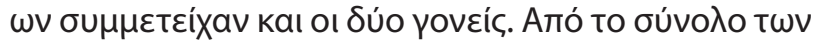

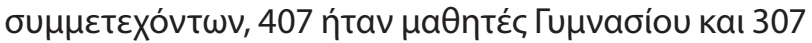

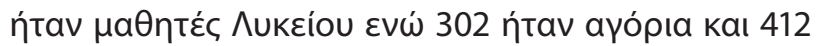
ńtav kopítoıa.

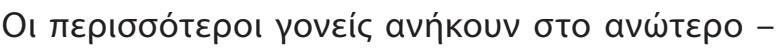

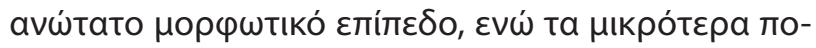

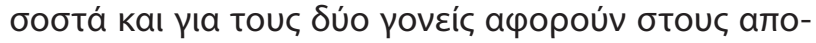

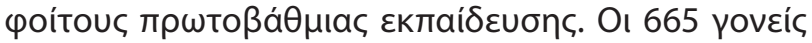

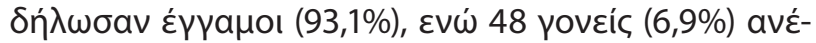




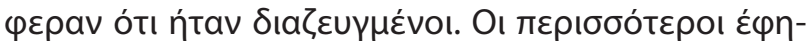

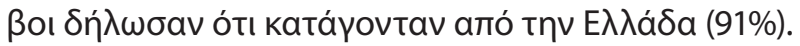

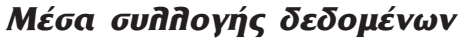

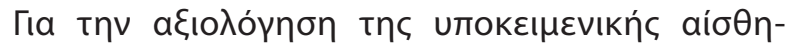

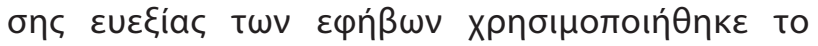

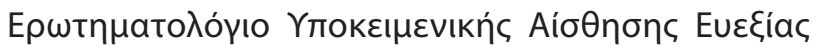

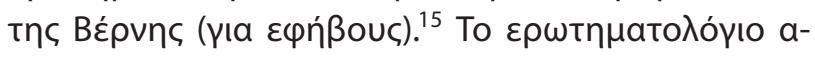

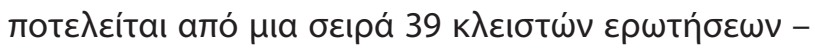

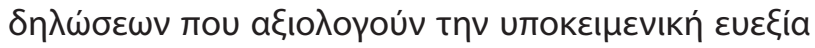

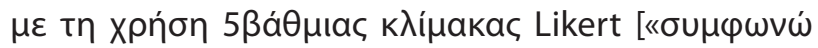

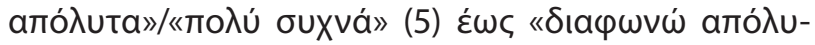

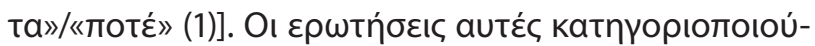

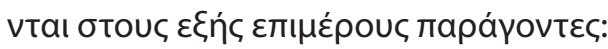

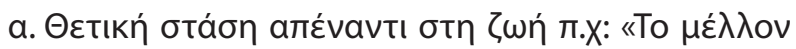

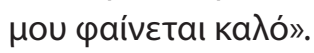

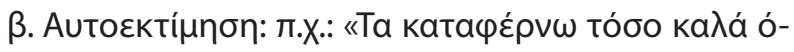

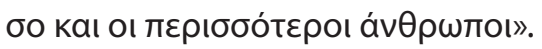

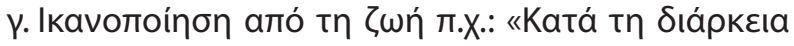

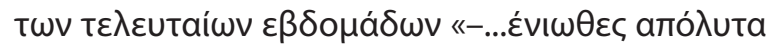

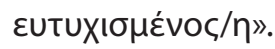

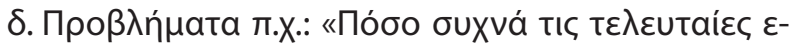

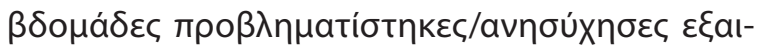

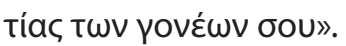

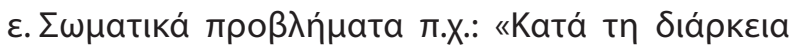

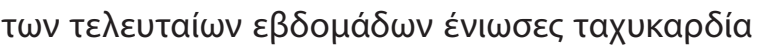

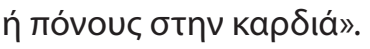

бт. КатаӨ пота пıа».

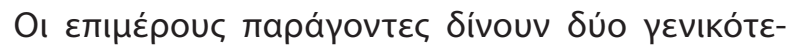

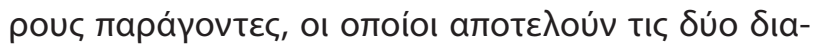

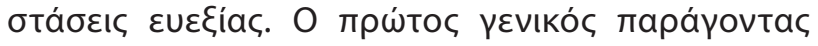

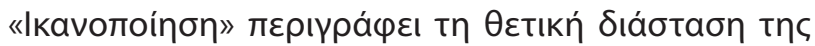

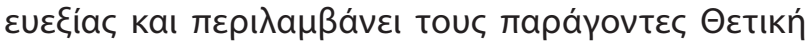

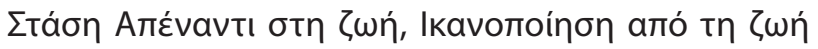

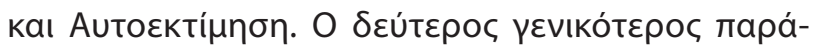

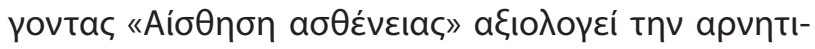

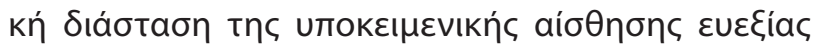

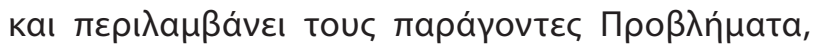

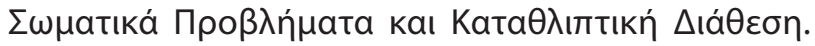

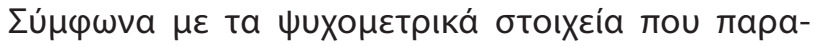

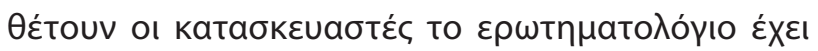

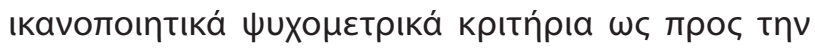

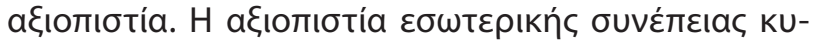

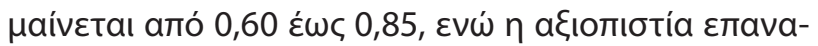

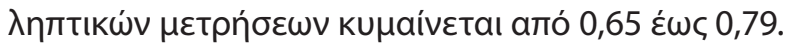

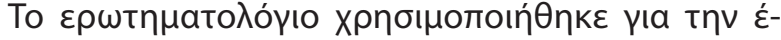

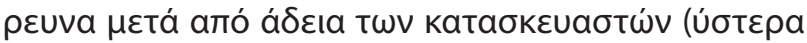

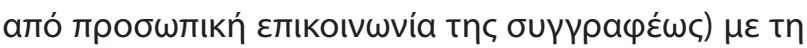

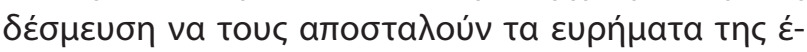

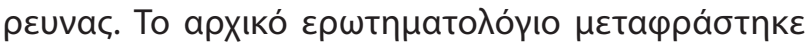

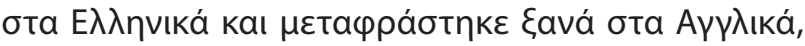

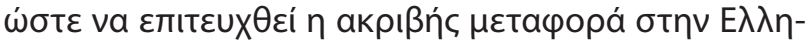

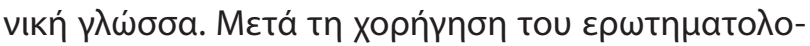

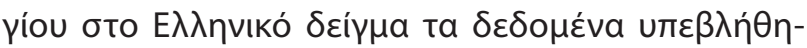

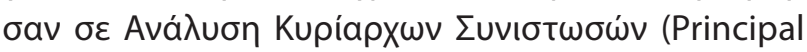

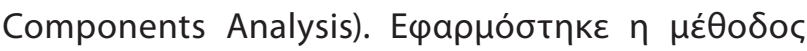

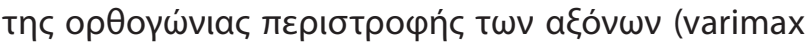

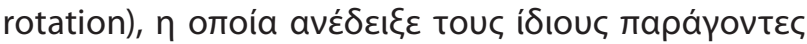

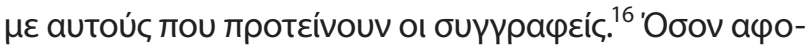

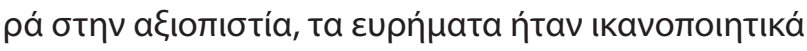

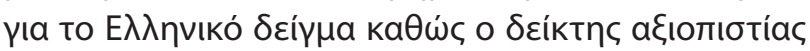

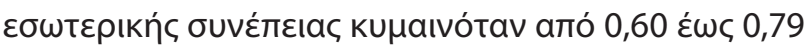

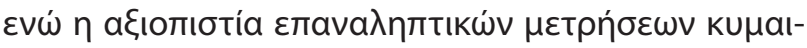

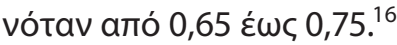

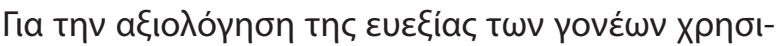
нотопиंӨпкаv:

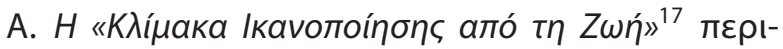

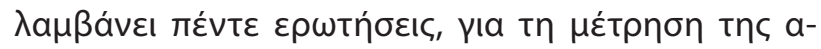

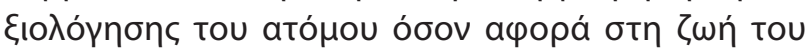

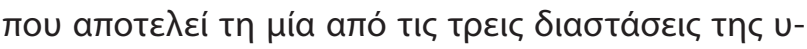

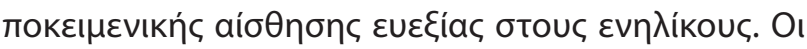

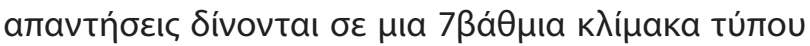

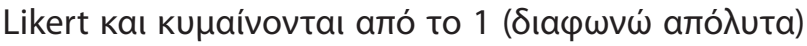

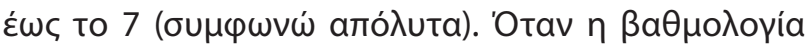

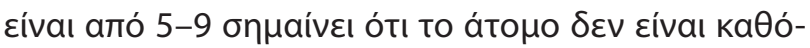

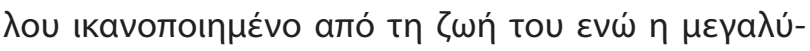

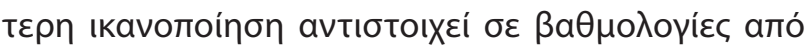

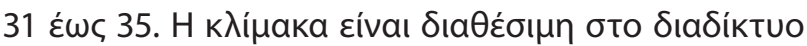

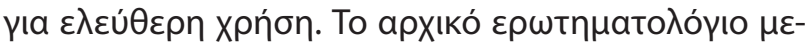

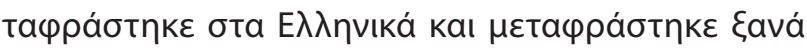

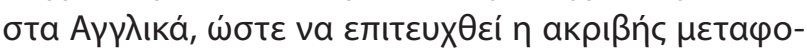

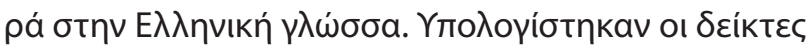

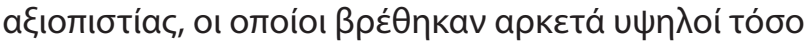

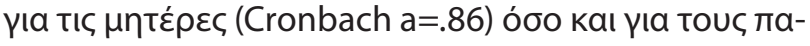

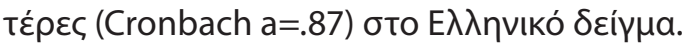

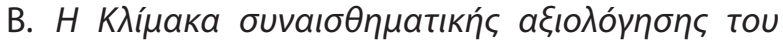

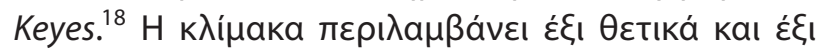

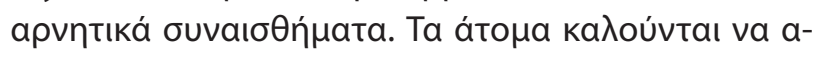

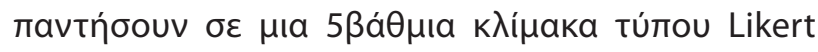

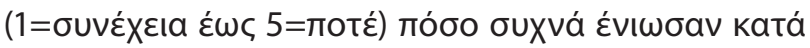

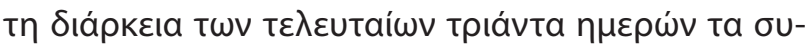

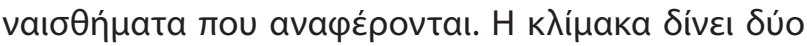




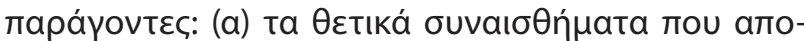

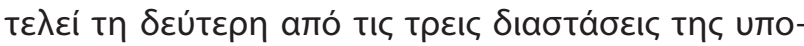

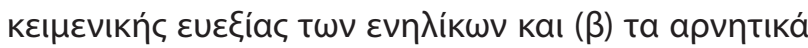

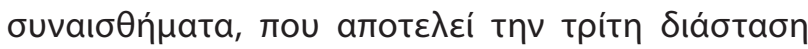

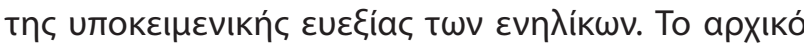

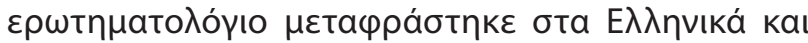

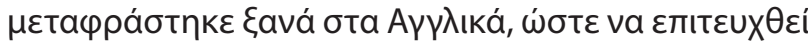

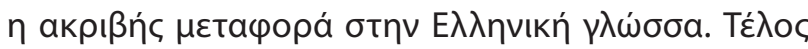

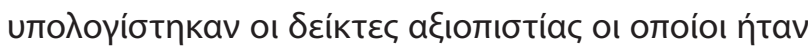

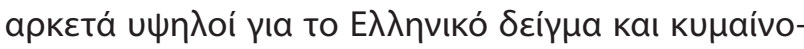

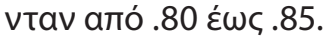

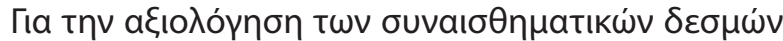

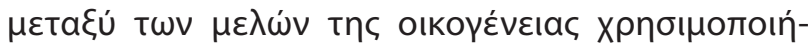

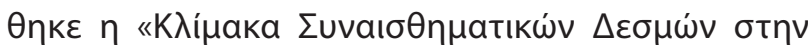

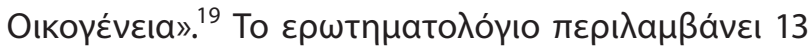

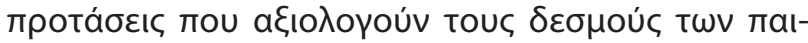

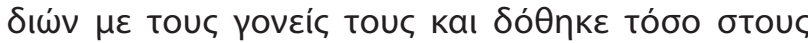

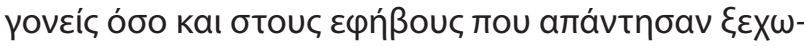

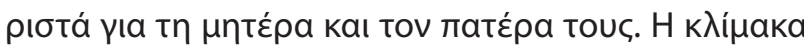

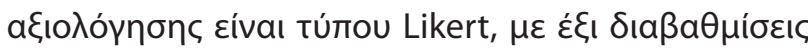

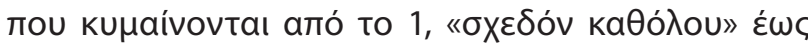

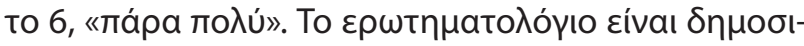

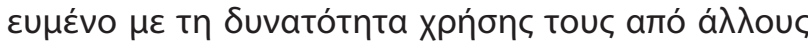

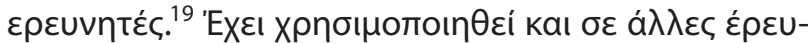

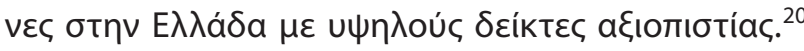

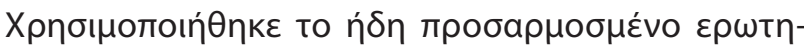

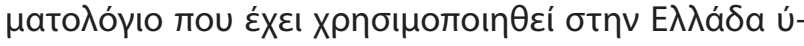

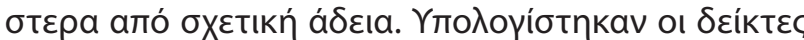

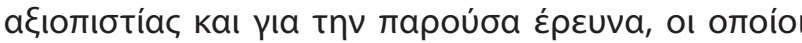

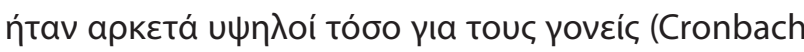

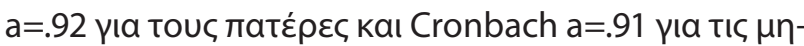

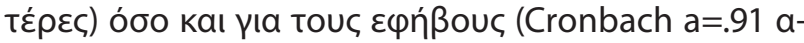

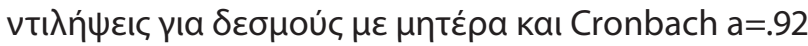

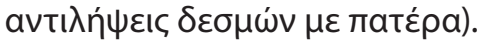

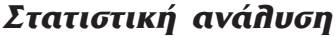

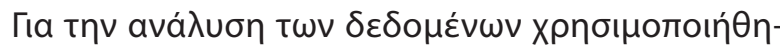

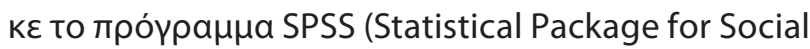

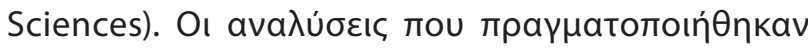

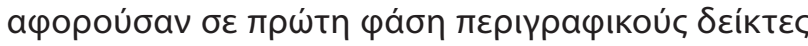

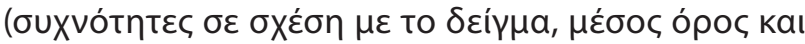

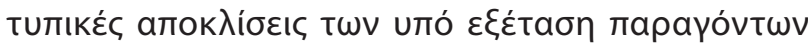

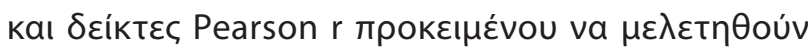

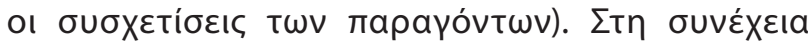

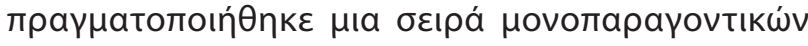

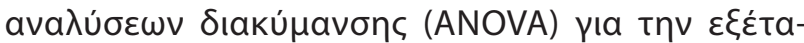

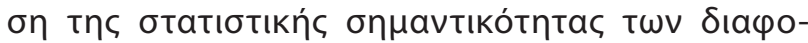

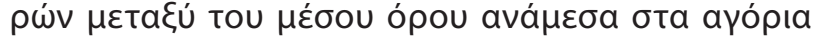

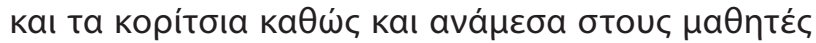

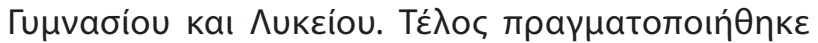

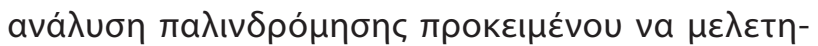
Ө

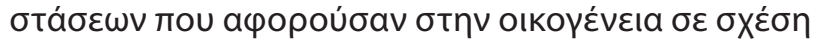

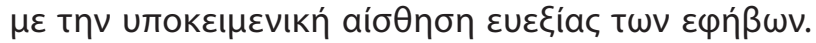

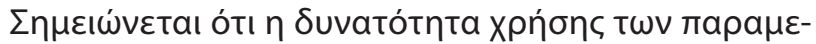

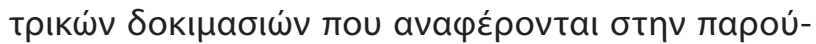

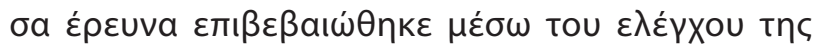

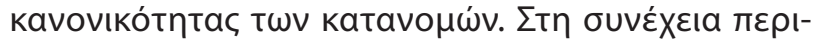

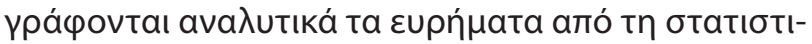

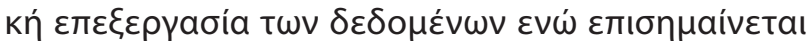

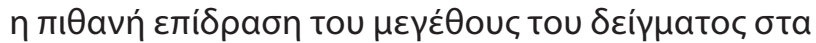

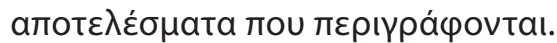

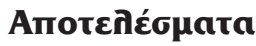

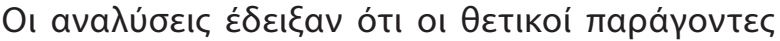

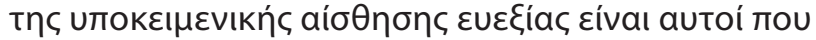

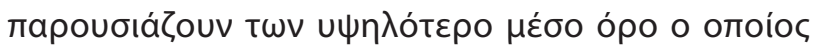

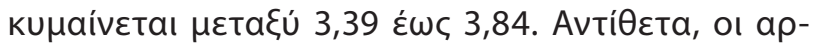

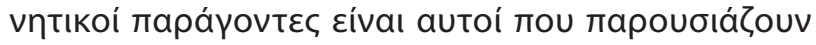

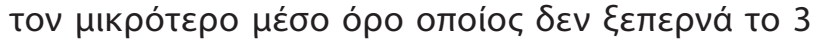
(ті́vakac 1).

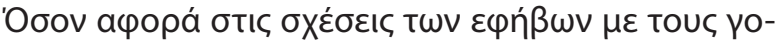

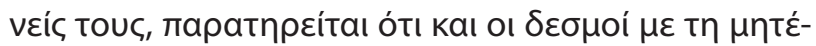

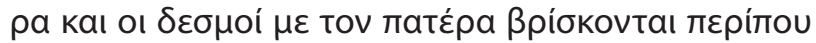

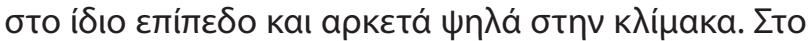

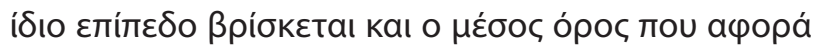

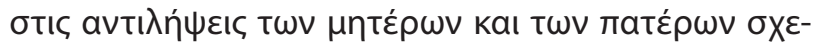

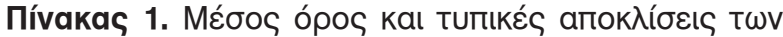

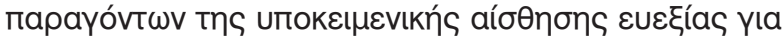

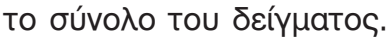

\begin{tabular}{|c|c|c|}
\hline 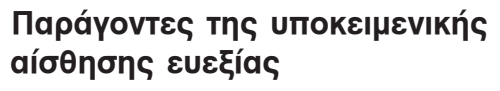 & MO & TA \\
\hline 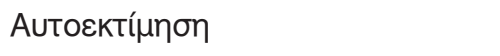 & 3,83 & 0,56 \\
\hline 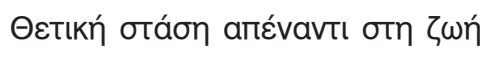 & 3,54 & 0,59 \\
\hline 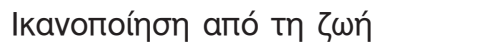 & 3,39 & 0,76 \\
\hline 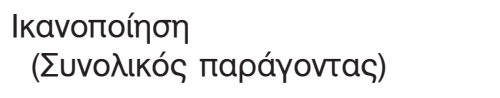 & 3,60 & 0,49 \\
\hline 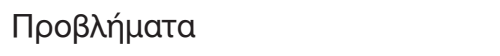 & 2,62 & 0,74 \\
\hline 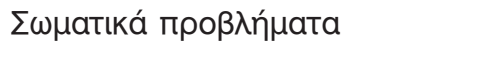 & 2,05 & 0,76 \\
\hline 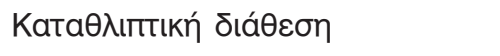 & 2,40 & 0,69 \\
\hline 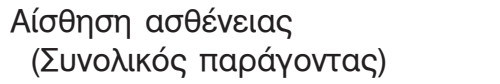 & 2,33 & 0,63 \\
\hline
\end{tabular}




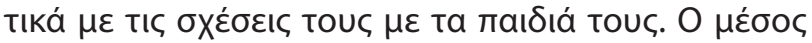

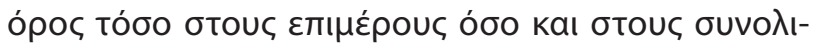

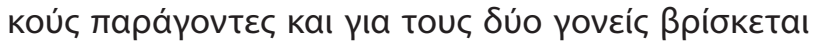

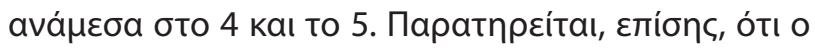

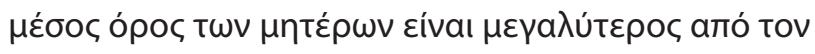

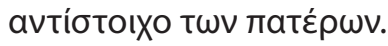

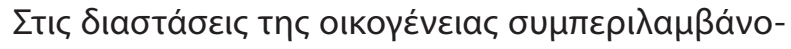

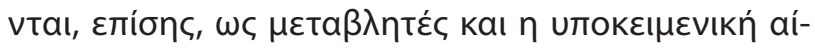

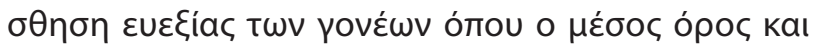

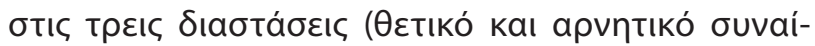

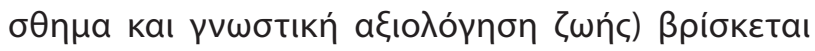

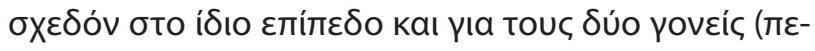

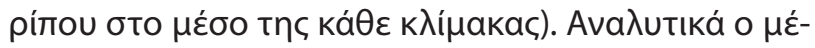

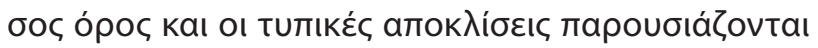
бтоv пívaкa 2.

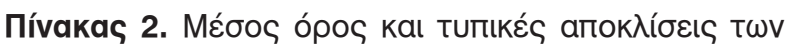

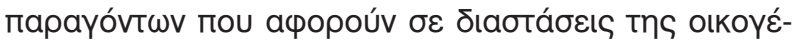

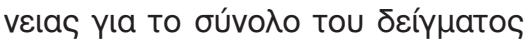

\begin{tabular}{|c|c|c|}
\hline 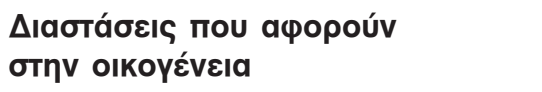 & MO & TA \\
\hline \multicolumn{3}{|l|}{ 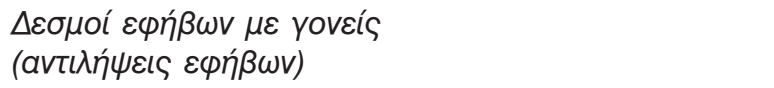 } \\
\hline$\Delta \varepsilon \sigma \mu o i ́ ~ \mu \varepsilon \mu \eta t \varepsilon ́ \rho a$ & 5,03 & 0,81 \\
\hline 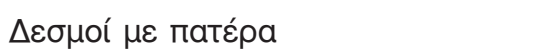 & 4,80 & 0,83 \\
\hline \multicolumn{3}{|l|}{ 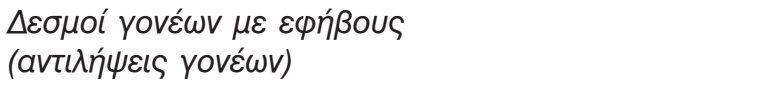 } \\
\hline 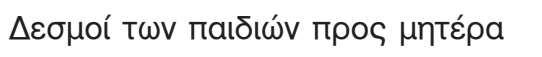 & 4,70 & 0,86 \\
\hline 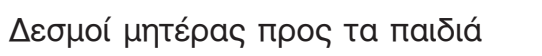 & 5,04 & 0,68 \\
\hline 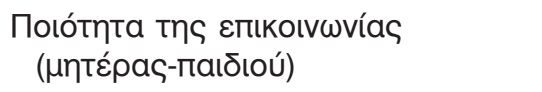 & 4,98 & 0,84 \\
\hline 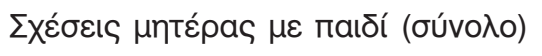 & 4,89 & 0,69 \\
\hline 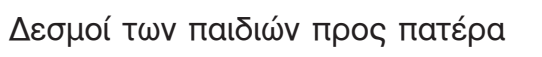 & 4,62 & 0,92 \\
\hline 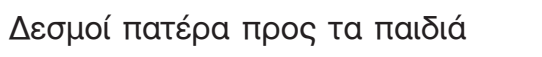 & 4,92 & 0,76 \\
\hline 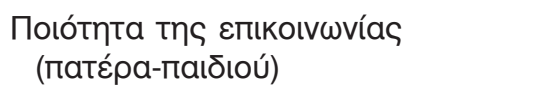 & 4,69 & 0,89 \\
\hline 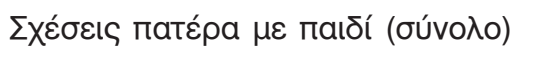 & 4,75 & 0,76 \\
\hline \multicolumn{3}{|l|}{ 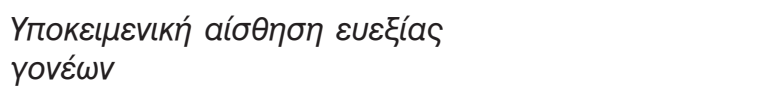 } \\
\hline 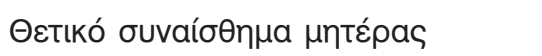 & 2,83 & 0,66 \\
\hline 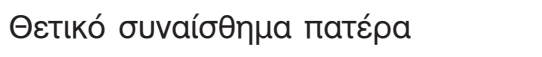 & 2,82 & 0,69 \\
\hline 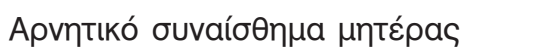 & 3,58 & 0,68 \\
\hline 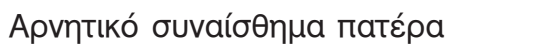 & 3,65 & 0,69 \\
\hline 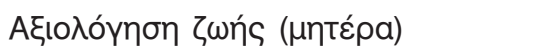 & 22,61 & 6,05 \\
\hline 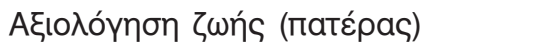 & 22,24 & 6,28 \\
\hline
\end{tabular}

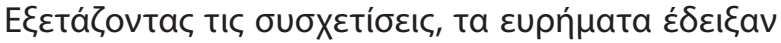

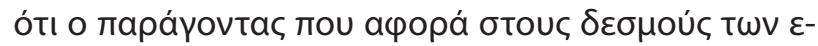

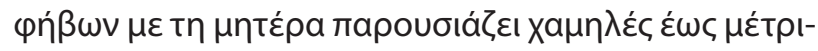

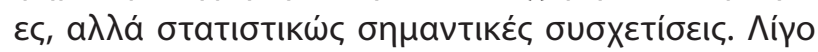

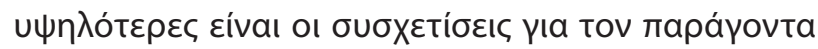

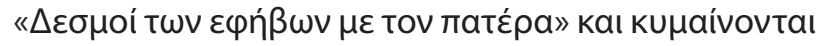

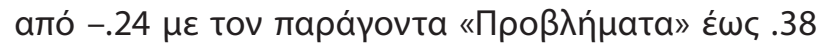

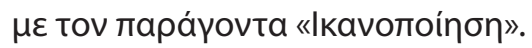

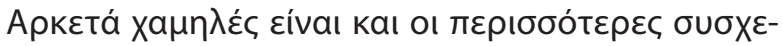

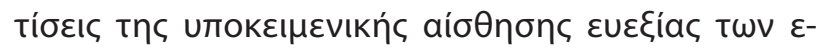

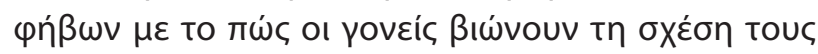

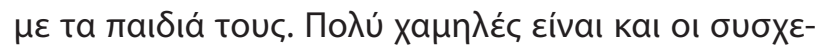

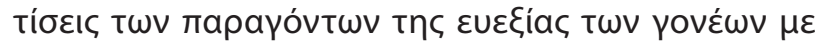

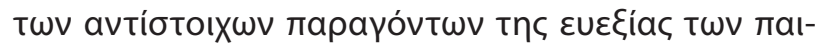

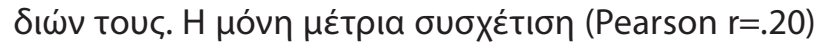

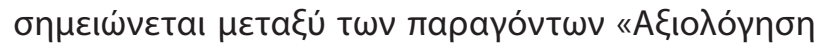

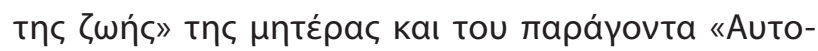

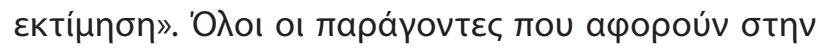

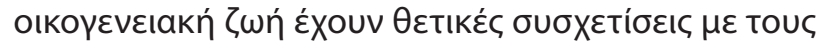

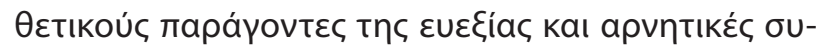

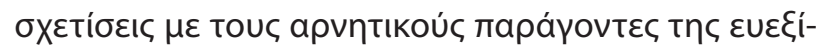

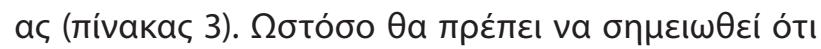

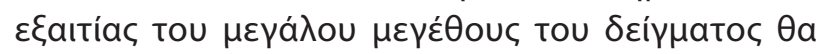

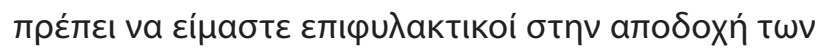

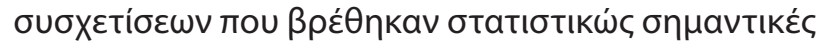

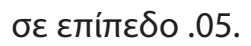

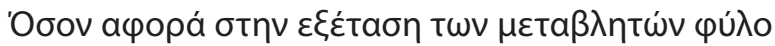

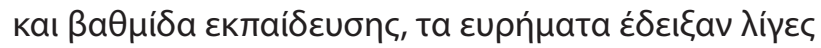

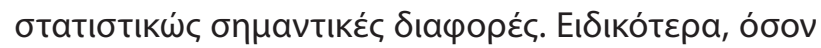

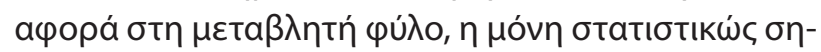

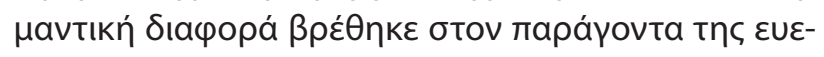

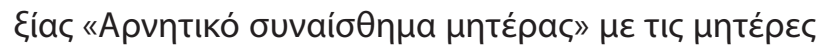

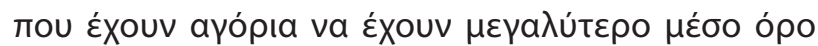

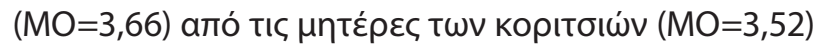

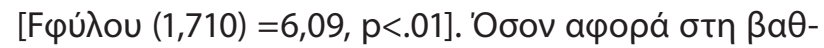

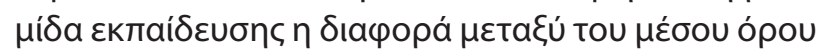

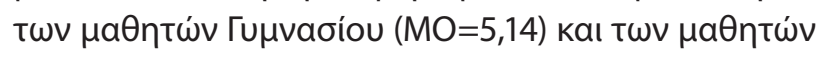

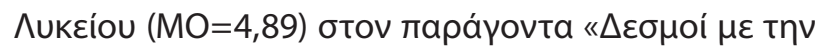

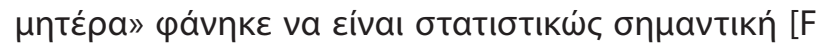

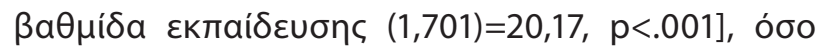

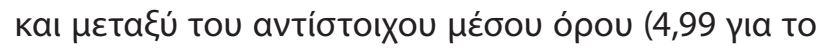

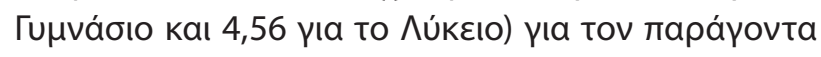

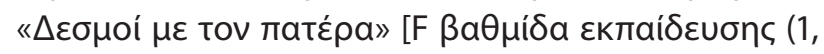
701) $=48,61, p<.001]$.

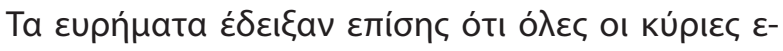

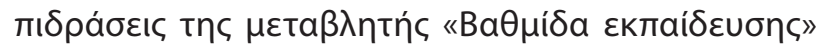

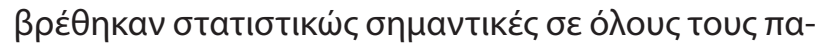




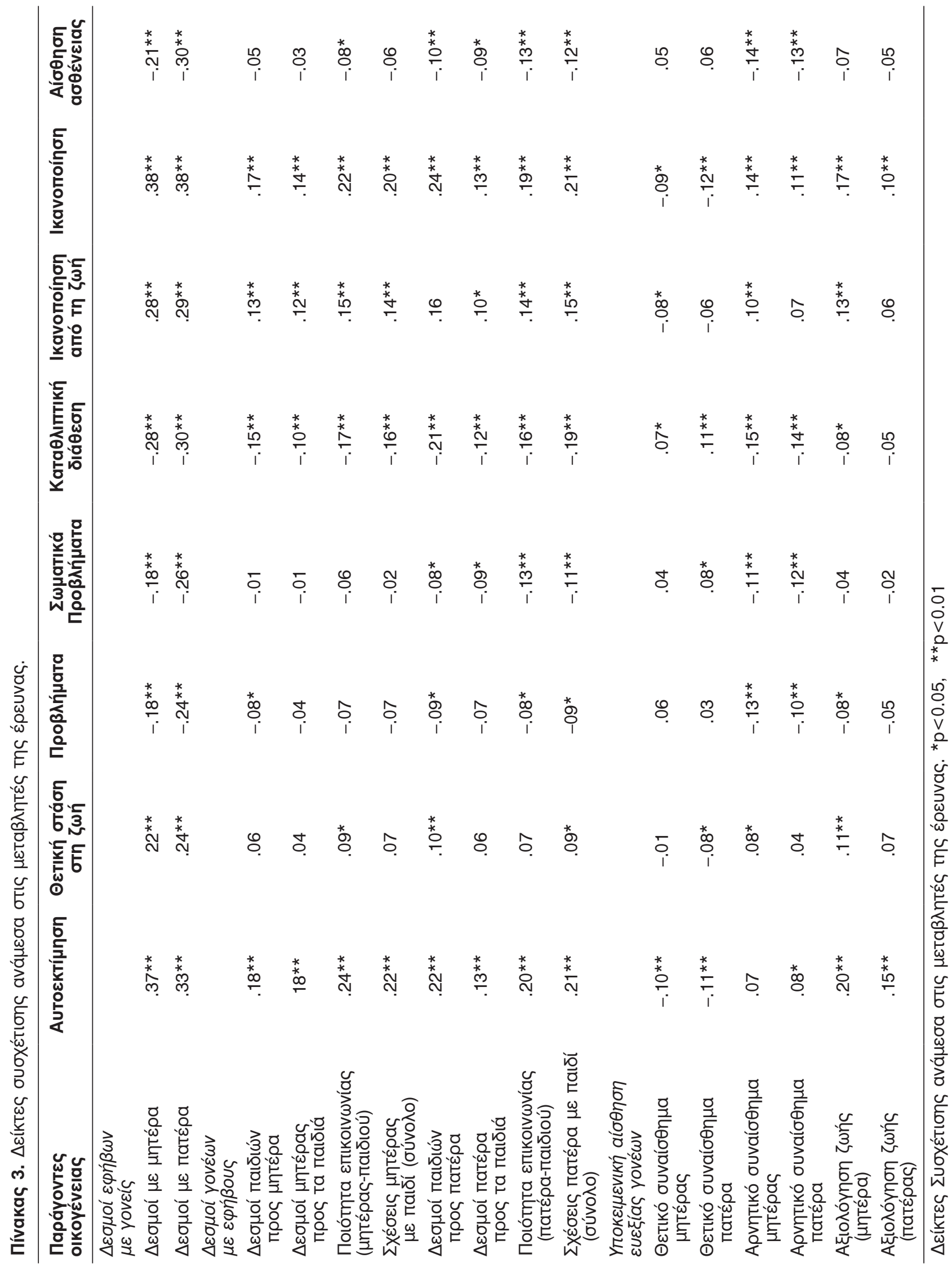




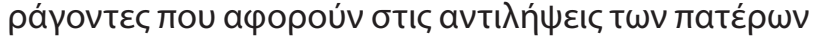

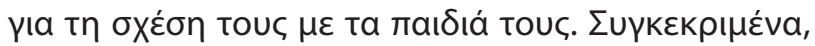

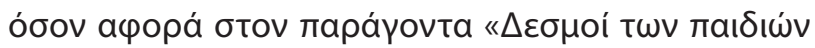

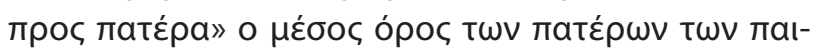

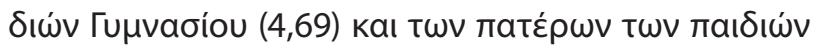

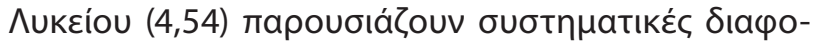

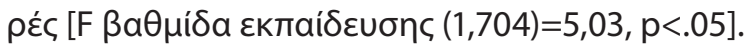

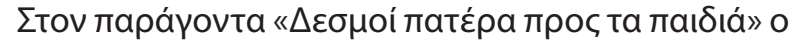

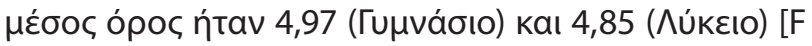

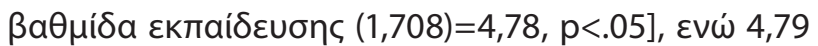

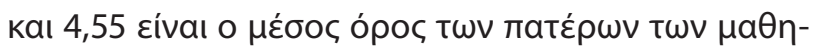

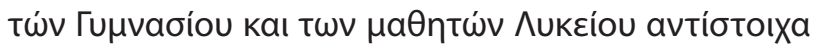

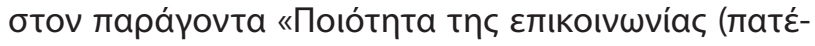

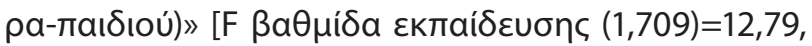

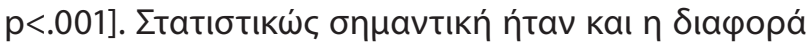

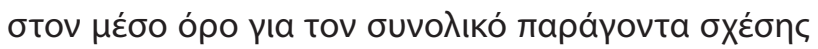

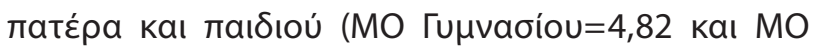

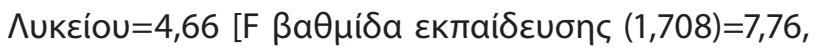
$\mathrm{p}<.01]$.

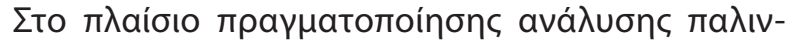

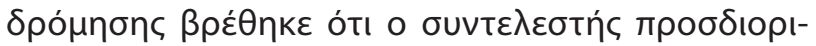

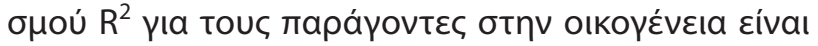

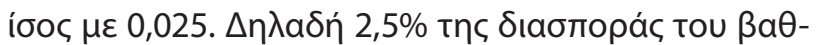

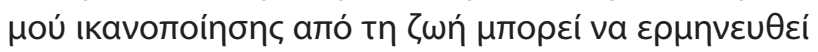

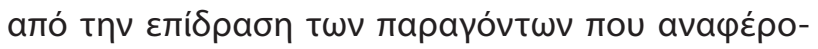

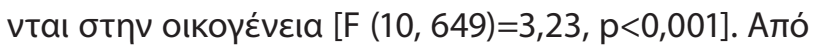

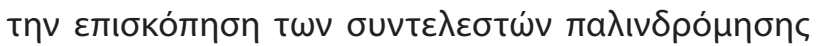

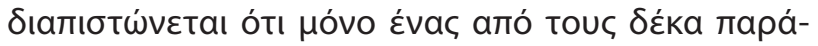

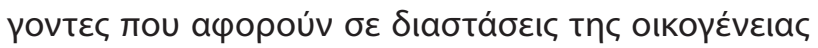

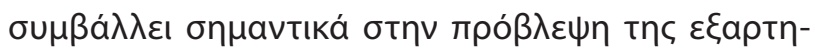

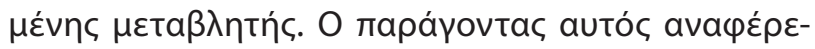

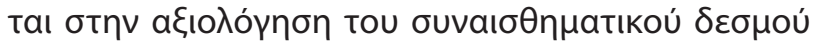

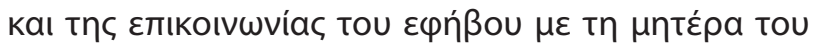
$(\beta=0,08, t=2,08, p<0,05)$. Autó on

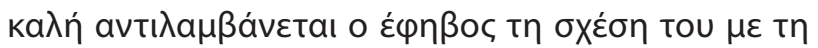

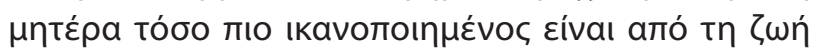

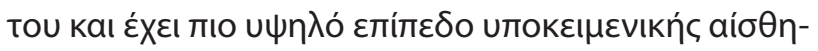

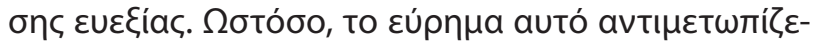

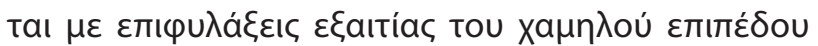

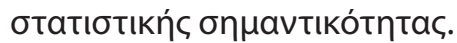

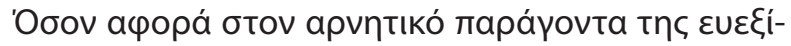

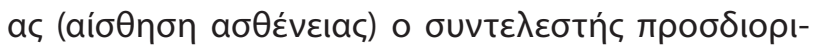

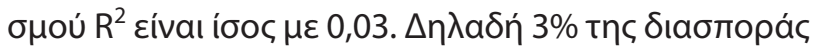

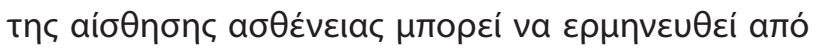

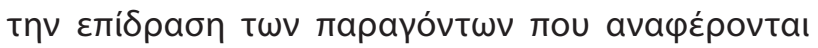

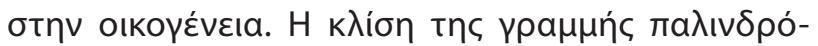

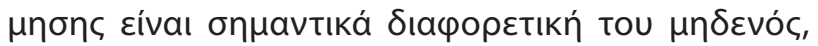

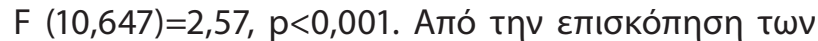

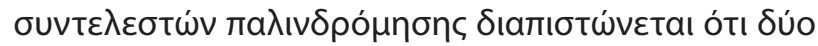

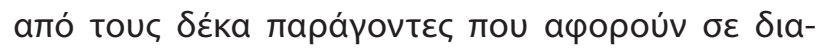

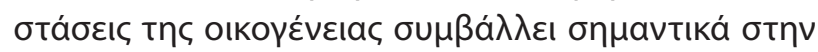

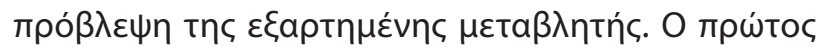

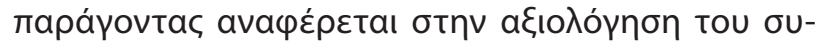

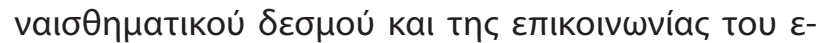

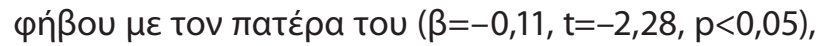

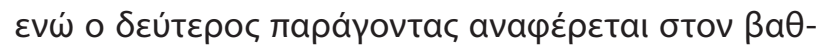

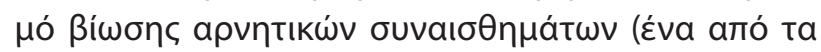

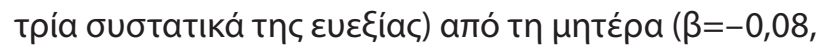

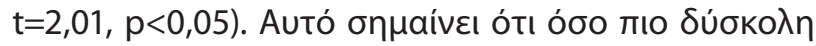

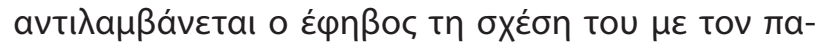

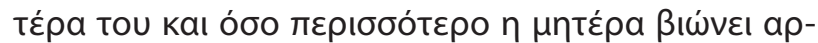

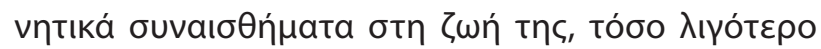

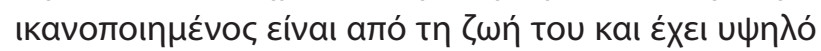

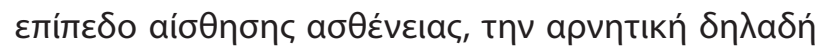

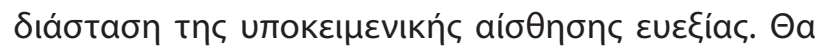

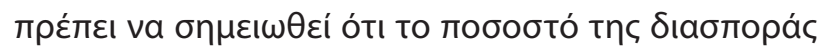

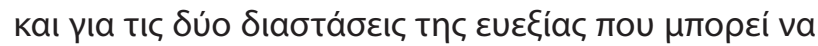

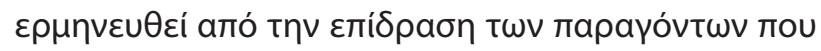

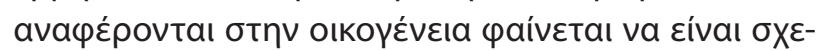

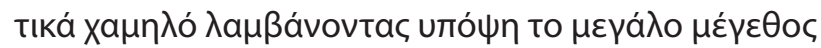

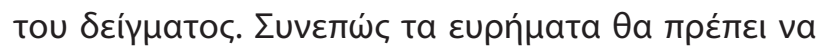

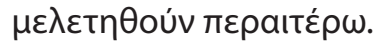

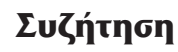

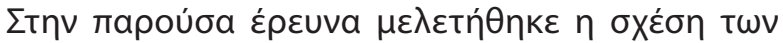

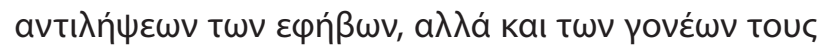

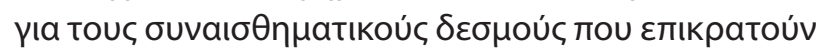

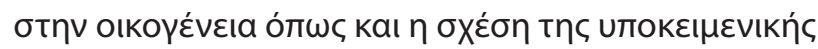

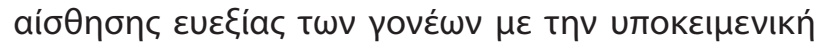

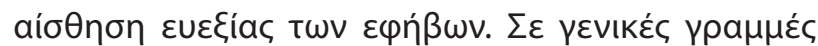

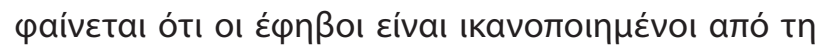

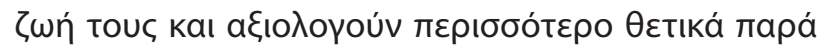

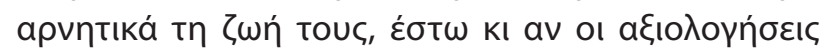

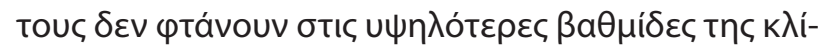

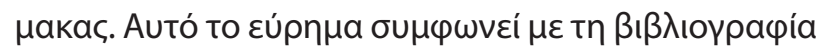

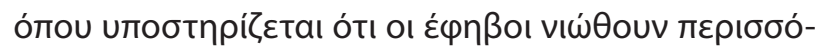

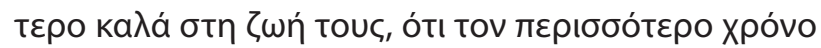

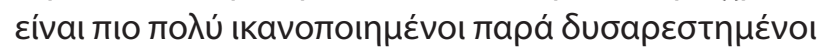

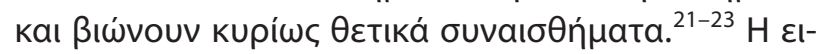

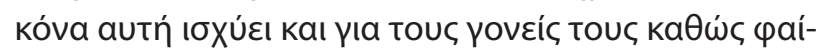

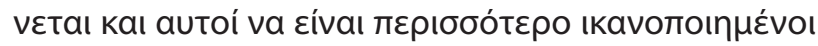

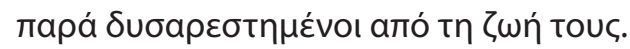

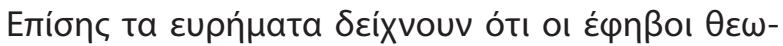

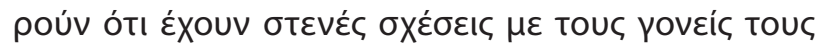




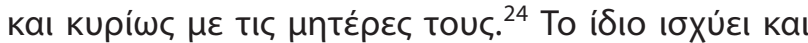

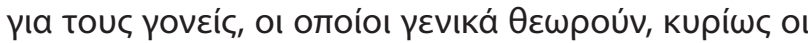

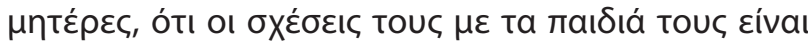

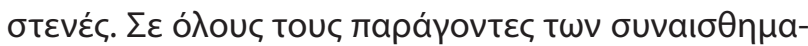

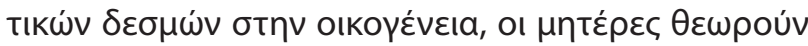

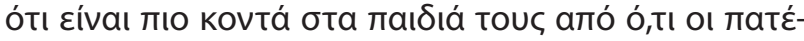

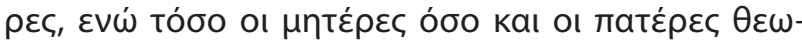

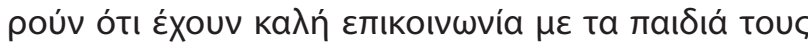

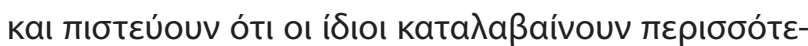

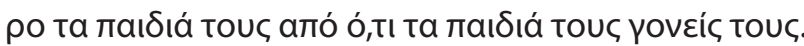

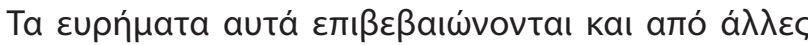

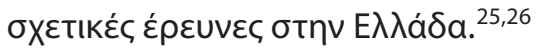

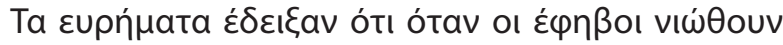

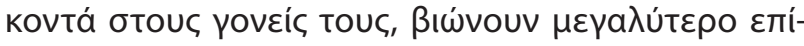

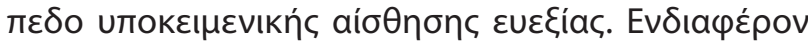

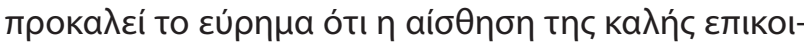

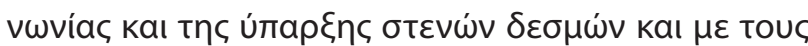

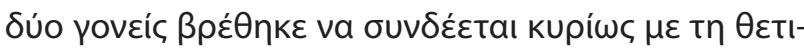

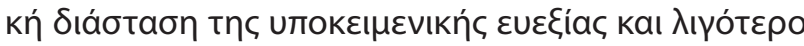

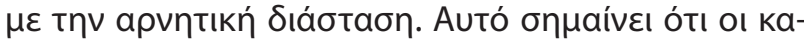

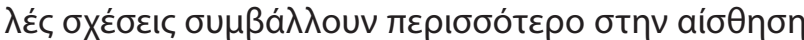

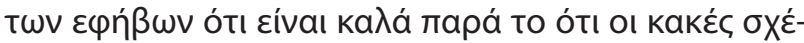

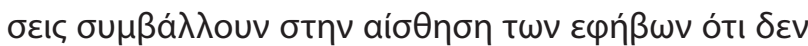

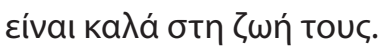

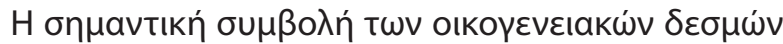

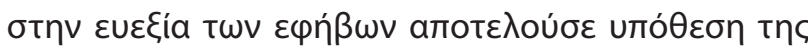

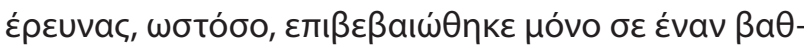
$\mu$

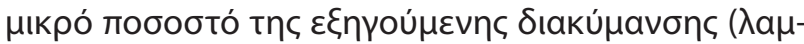

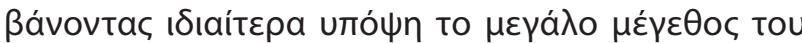

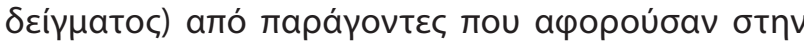

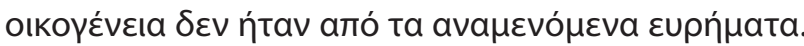

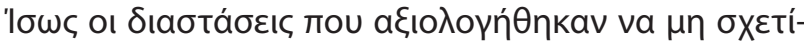

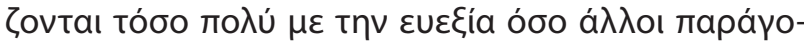

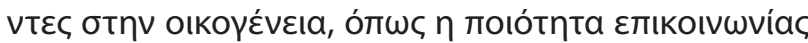

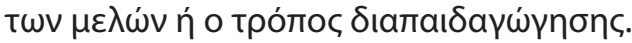

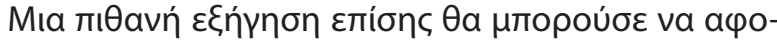

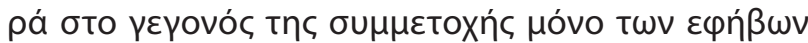

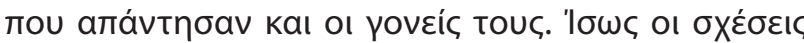

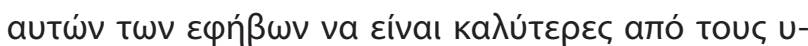

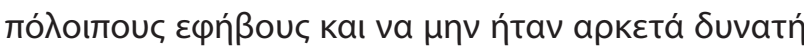

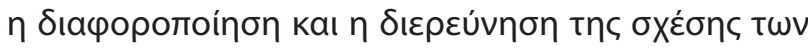
$\mu \varepsilon \tau a \beta \lambda \eta \tau \omega ́ v$.

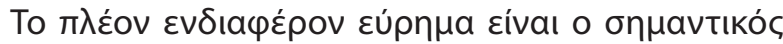

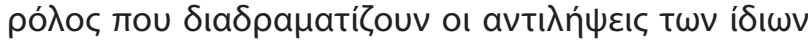

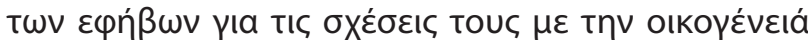

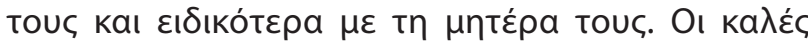

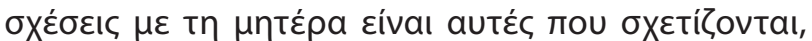

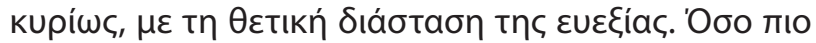

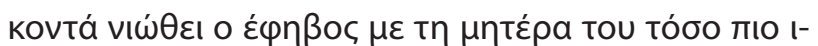

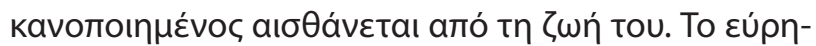

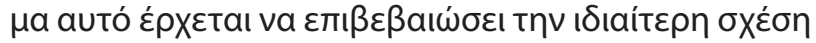

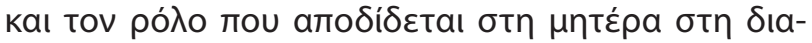

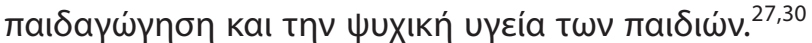

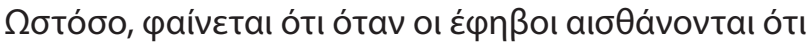

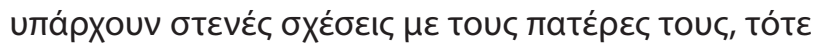

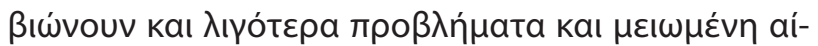
бӨnon aбӨźvelac.

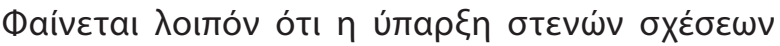

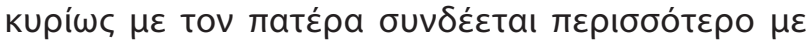

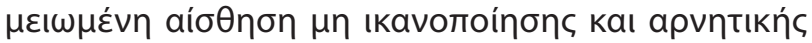

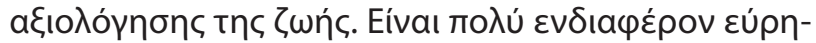

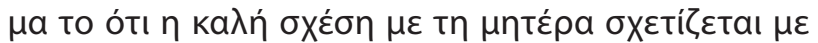

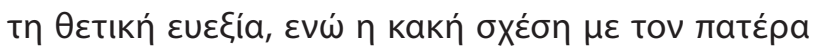

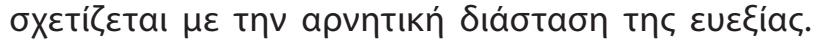

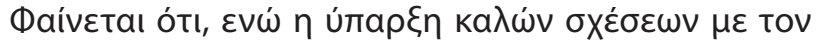

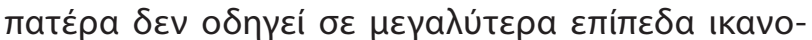

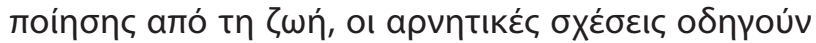

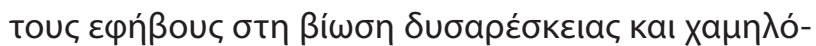

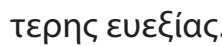

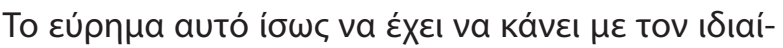

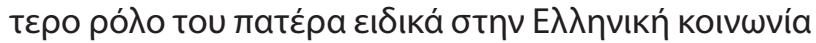

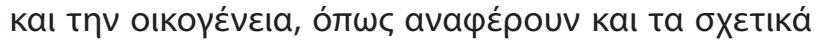

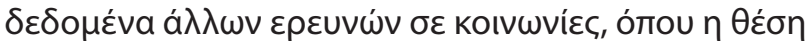

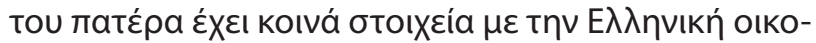

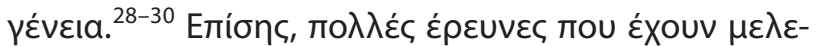

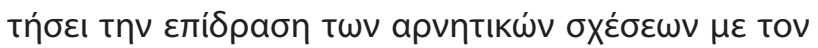

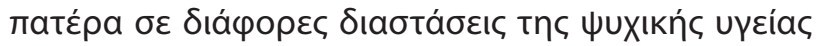

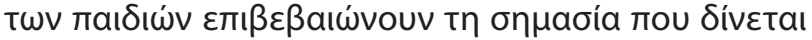

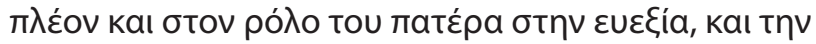

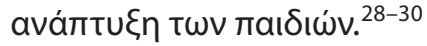

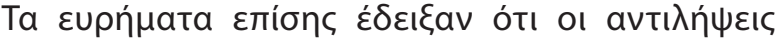

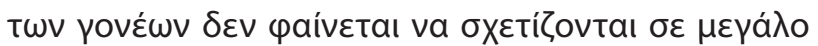

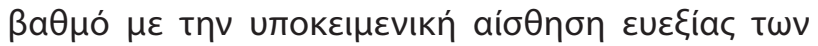

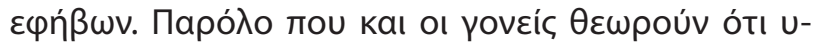

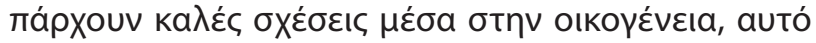

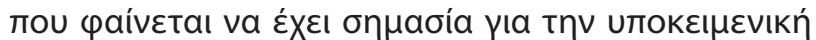

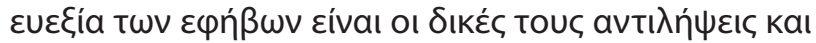

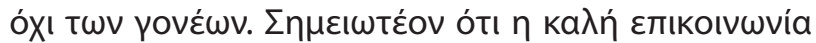

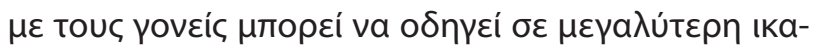

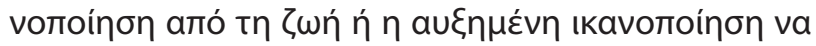

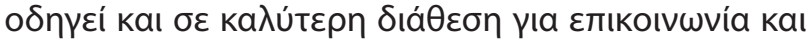

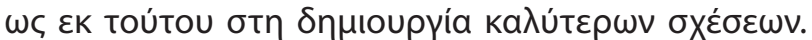

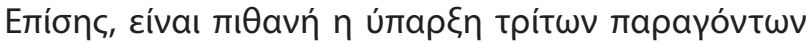




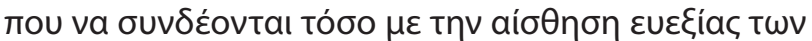

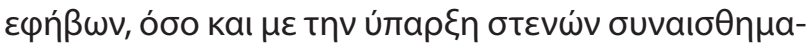

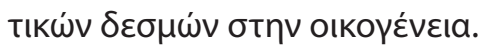

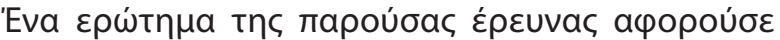

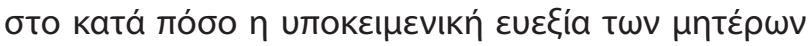

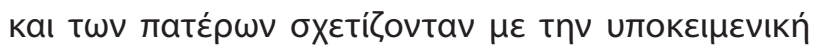

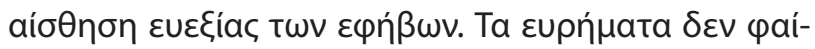

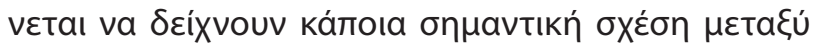

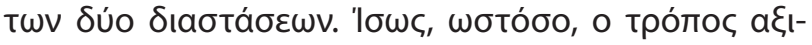

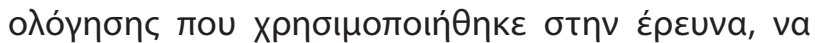

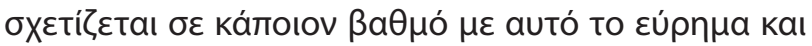

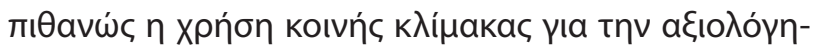

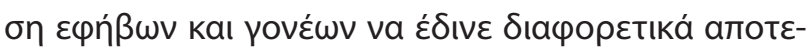

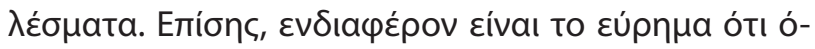

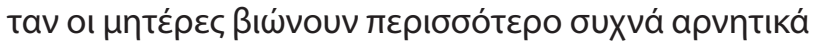

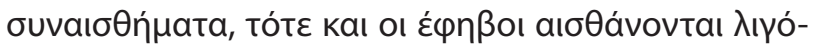

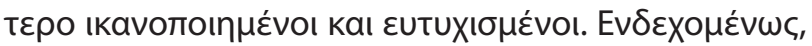

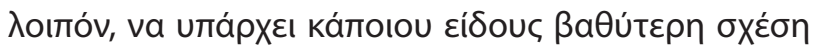

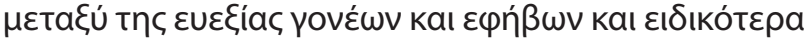

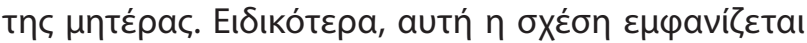

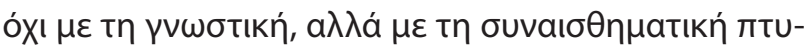

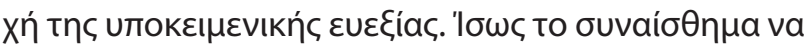

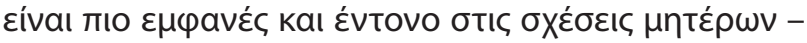

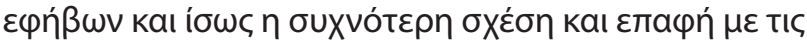

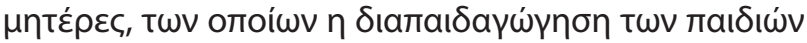

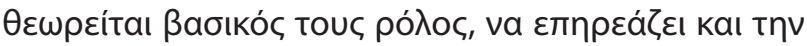

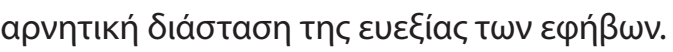

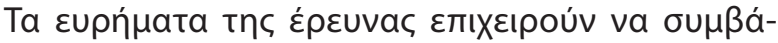

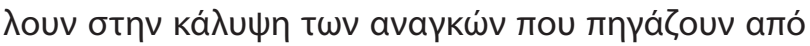

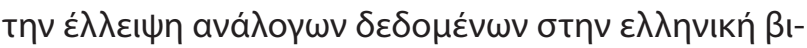

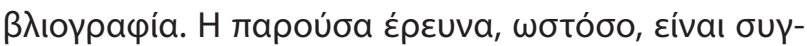

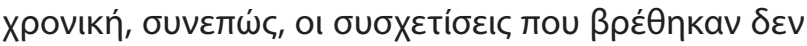

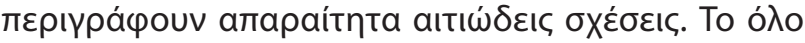

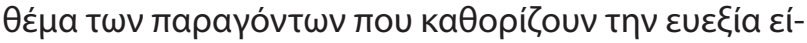

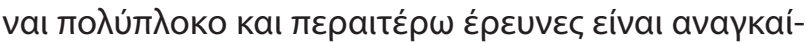

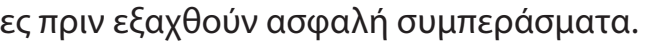

\title{
The role of the family in adolescents' subjective well-being
}

\author{
A. Lampropoulou \\ Department of Psychology, University of Athens, Athens, Greece
}

Psychiatriki 2018, 29:172-182

The goal of this research is to study the relation between adolescents' subjective well-being with: (a) their perceptions regarding their relationship with their parents, (b) the corresponding perceptions of their parents and (c) parents' subjective well-being. The participants of this study were 714 adolescents attending the two last grades of Junior High School (Gymnasium) and the two last grades of High School (Lyceum) as well as their parents from schools of the broader area of Athens randomly selected. The following questionnaires were used for data collection from the adolescents who participated in the study: (a) The assessment of subjective well-being was based on the Berne Questionnaire of Subjective Well-Being/ Youth Form that provides the following factors: Positive attitude towards life, Self-esteem, Satisfaction from life, Problems, Somatic complaints and Depressive mood. The first three factors are included in a general factor called Satisfaction which is the positive aspect of subjective well-being (according to the suggested structure of the concept in the relevant literature) while the remaining three are included in a second general factor called III-being which is the negative aspect of subjective well-being, and (b) the assessment of family relationships was based on the "Affectual solidarity in the family scale" (which was also administered to parents). In addition, parents completed the questionnaires "Satisfaction from Life scale" and the "Assessment of the emotional satisfaction from life-Keyes scale" for assessing their level of subjective well-being. Finally, information on demographics was also collected. Statistical analysis of the data included descriptive and deductive methods in order to examine the relationship and the interaction of the variables under research. Findings revealed several interesting relationships. In particular 
the results showed that the better the relationships are perceived by the adolescents the more satisfied they feel from their lives. In particular, the good relationships with the mother strengthen the positive dimension of subjective well-being while the good relationships with the father decrease the negative dimension of subjective well-being. Findings also suggest no significant relation among parents' perceptions and their subjective well-being with the well-being of the adolescents. The results of the research provide the guidelines for further research on the role of the family in adolescents' subjective well-being while they also allow for a better understanding of the way adolescents' subjective well-being is related to various variables in order to further enhance it through appropriate interventions.

Key words: Adolescence, subjective well-being, family, satisfaction from life.

\section{BıBAtoypacpía}

1. Brotherson SE, Yamamoto T, \& Acock AC. Connection and communication in father-child relationships and adolescent child well-being. Fathering 2003, 1:191-214, doi: 10.3149/ fth.0103.191

2. Diener E. Subjective well-being: The science of happiness and a proposal for a national index. Am Psychol 2000, 55:34-43, doi: 10.1037/0003-066X.55.1.34

3. Karademas EC. Self-efficacy, social support and well-being: The mediating role of optimism. Pers Individ Dif 2006, 40:1281-1290, doi: 10.1016/j.paid.2005.10.019

4. Karademas EC. Subjective well-being, demographic and intrapersonal correlates. $\Psi_{u}$ Xo ${ }^{\prime}$ oyía in press

5. Behnke AO, MacDermid SM. Family well-being. A Sloan work and family encyclopedia entry 2004, Available from: http:// www. bc.edu/bc_org/

6. Hann CM. The relationship between certain family variables and the psychological well-being of black adolescents, 2005, Doctoral dissertation, University of the Free State, Bloemfontein Available from: http://www.scholar.ufs.ac.za

7. Hetherington EM. Social support and the adjustment of children in divorced and remarried families, Childhood 2003, 10(2): 217-36, doi: 10.1177/0907568203010002007

8. Amato PR, \& Afifi TD. Feeling caught between parents: adult children's relations with parents and subjective well-being, J Marriage Fam 2006, 68:222-235, doi: 10.1111/j.17413737.2006.00243

9. Rask K, Astedt-Kurk P, Paavilainen E, \& Laippala P. Adolescent subjective well-being and family dynamics. Scand J Caring Sci 2003, 17:129-138, doi: 10.1037/0033-2909.132.2.249

10. Ryff CD, Singer BH, Wing E, \& Love GD. Elective affinities and uninvited agonies: mapping emotion with significant others onto health. In: Ryff CD. \& Singer BH (eds) Emotion, Social Relationships, and Health, New York, NY: Oxford University Press, 2001:133-175, doi: 10.1093/acprof:oso/9780 195145410.003.0005

11. Johnson LD, O'Malley PM, Bachman JG. National survey results on drug use from the monitoring the future study, 1975-2000: Vol. 1. Secondary school students (NIH Publication No. 2000). Rockville, MD: National Institute on Drug Abuse. 2001 Available from: http://www.monitoringthefuture.org/pubs/monographs/ vol1_2002.pdf
12. Ryan RM, Deci EL. On happiness and human potentials: A review of research on hedonic and eudaimonic well-being. Annu Rev Psychol 2001, 52:141-166, doi: 10.1146/annurev. psych.52.1.141

13. Flouri E. Subjective well-being in midlife: the role of involvement of and closeness to parents in childhood. $J$ Happiness Stud 2004, 5: 335-358, doi: 10.1023/b:johs.0000048461.21694.92

14. Joronen K, Astedt-Kurki P. Familial contribution to adolescent subjective well-being. Int J Nurs Stud 2005, 11:125-133, doi: 10.1111/j.1440-172X.2005.00509.x

15. Grob A, Luthi R, Kaiser F, Flammer A, Mackinnon A, Wearing A. Berner fragebogen zum wohlbefinden jugendlicher (BSW-Youth Form), Diagnostica 1991, 37:66-75 Available from: http://edoc. unibas.ch/dok/A5255072

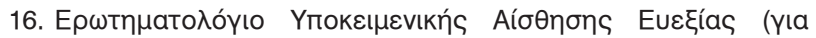

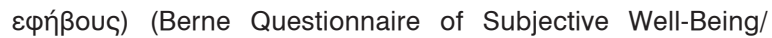

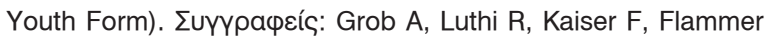

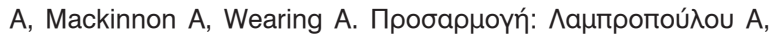

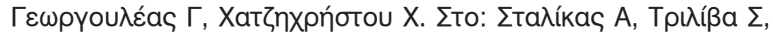

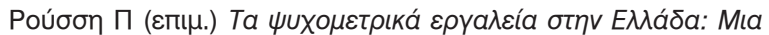

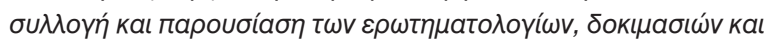

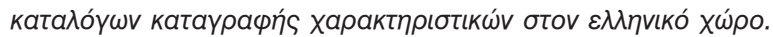
AӨńva, Пєסío. 2012:713

17. Diener E, Emmons RA, Larsen RJ, Griffin S. The Satisfaction with Life Scale. J Pers Assess 1985, 49:71-75, doi: 10.1037/10403590.5.2.164

18. Keyes CLM, Social well-being. Soc Psychol Q 1998, 61:121-140, doi: $10.2307 / 2787065$

19. Gronvold RL. Measuring affectual solidarity. In: Mangen D, Vern J. Bengtson L, Landry P (eds) The measurement of intergenerational relations. Beverly Hills, CA: Sage, 1988:74-97 Available from: http://psycnet.apa.org/record/1988-97094-004

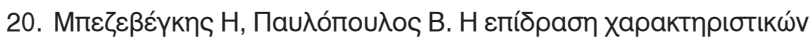

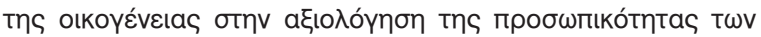

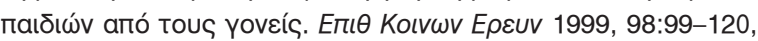
doi: $10.12681 / \mathrm{grsr} .744$

21. Gilman R, Huebner S. A review of life satisfaction research with children and adolescents. Sch Psychol Q 2003, 18:192-205, doi: 10.1521/scpq.18.2.192.21858

22. Karademas EC. Positive and negative aspects of well-being: Common and specific predictors. Pers Individ Dif 2007, 43:277287, doi: 10.1016/j.paid.2006.11.031 


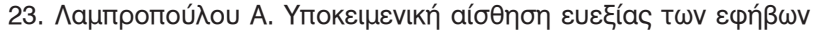

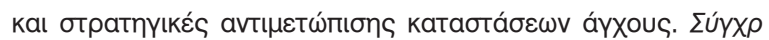

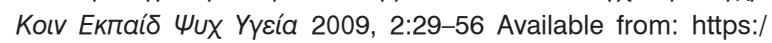
aigaion.org/skepsy

24. Lampropoulou A, Georgouleas G, Hatzichristou C. Subjective well-being and coping strategies of Greek adolescents. Poster presented at IXth Conference of European association for research on Adolescence, Porto 2004

25. Georgouleas G, Besevegis E. Adolescents' views of their rela-

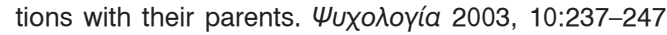

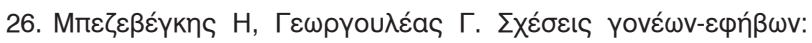

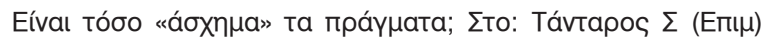

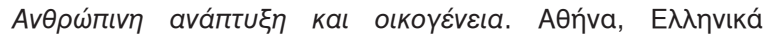
Гра́ $\mu$ ata, 2004:103-12

27. Li-xing Z, Guang-fang T, The influential factors on quality of life in children. Chin J Clin Psychol 2001, 9:105-107 Available from: https://caod.oriprobe.com/articles/3262155

28. Flouri E, Buchanan A. Childhood predictors of labor force participation in adult life. JFEI 2002, 23:101-120, doi: 10.1023/ A: 1015776832505
29. Shek DTL. The relation of parental qualities to psychological well-being, school adjustment and problem behavior in Chinese adolescents with economic disadvantage. Am J Fam Ther 2002, 30:215-230, doi: 10.1080/019261802753577548

30. Maccoby EE. Parenting and its effects on children: On reading and misreading behavior genetics. Annu Rev Psychol 2000, 51:1-27, doi: 10.1146/annurev.psych.51.1.1

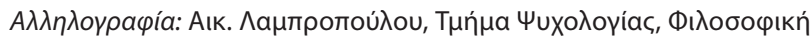

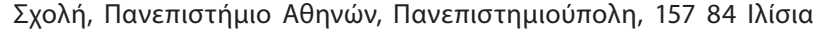

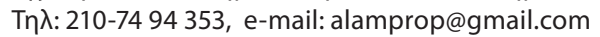

